\title{
DESCRIPCIÓN DE LA CALIDAD FISICOQUÍMICA DE LOS RESIDUOS DE SABILA (Aloe vera) (L.) Burm. f.
}

\author{
DESCRIPTION OF THE PHYSICOCHEMICAL QUALITY OF SABILA RESIDUES \\ (ALOE VERA) (L.) BURM. F..
}

\author{
Guillermo Andrés Torres-Gómez'; Johan Sebastián Marulanda-Raigoso²; \\ Rigoberto Villa-Ramírez ${ }^{3}$ \\ ${ }^{1}$ Facultad Ciencias Agroindustriales, Programa Tecnología Agropecuaria, Universidad del Quindío. \\ aggomest@uqvirtual.edu.co \\ ${ }^{2}$ Facultad Ciencias Agroindustriales, Programa Tecnología Agropecuaria, Universidad del Quindío. \\ jsmarulandar@uqvirtual.edu.co \\ ${ }^{3}$ Facultad Ciencias Agroindustriales, GRUPO GICAP, Universidad del Quindío. \\ rivilla@niquindio.edu.co - https://orcid.org/0000-0002-4355-2230 \\ *Correspondencia del Autor: Rigoberto Villa Ramírez, correo electrónico: rivilla@niquindio.edu.co
}

\section{RESUMEN}

Esta investigación permitió realizar un análisis bromatológico a los residuos sólidos orgánicos generados en la planta procesadora de Aloe Vera de la empresa Sabimax loe S.A.S ubicada en el municipio de Montenegro Quindío, los cuales son el producto desechado de los procesos agroindustriales que realizan. Se realizaron pruebas bromatológicas como porcentaje de humedad, cenizas, grasa, fibra, actividad de agua y $\mathrm{pH}, \mathrm{y}$. Los análisis realizados a los residuos sólidos orgánicos determinaron que el porcentaje de humedad es del 92,4\%. Las demás pruebas fisicoquímicas realizadas sobre materia seca indicaron que el $0,43 \%$ corresponde a cenizas, el $39,6 \%$ a fibra, el $1,25 \%$ a extracto etéreo o grasa y el $0.95 \%$ es de actividad de agua, así mismo; se determinó un $\mathrm{pH}$ de 4,98 . Por lo que se encontraron en un promedio dentro de los rangos teóricos.

Palabras clave: Sábila; usos; análisis; desechos orgánicos; aloe vera.

Cómo citar:

Torres-Gómez, Guillermo Andrés; Marulanda-Raigoso, Johan Sebastián; Villa-Ramírez, Rigoberto (2020) DESCRIPCIÓN DE LA CALIDAD FISICOQUÍMICA DE LOS RESIDUOS DE SABILA (Aloe vera) (L.) Burm. f. Revista de Investigaciones Universidad del Quindio, vol. 32(1), pp.16-21. https://doi.org/10.33975/riuq.vol32n1.271 


\begin{abstract}
This investigation allowed to carry out a bromatological analysis of the organic solid waste generated in the Aloe Vera processing plant of the Sabimax loe S.A.S company located in the municipality of Montenegro Quindío, which is the product discarded from the agroindustrial processes they perform. Bromatological tests were performed as percentage of humidity, ash, fat, fiber, water activity and $\mathrm{pH}$, and. The analyzes performed on organic solid waste determined that the percentage of humidity is $92.4 \%$. The other physicochemical tests performed on dry matter indicated that $0.43 \%$ corresponds to ashes, $39.6 \%$ to fiber, $1.25 \%$ to ethereal or fat extract and $0.95 \%$ is water activity, likewise; a $\mathrm{pH}$ of 4.98 was determined. So they were found in an average within the theoretical ranges.
\end{abstract}

Keywords: Aloe; uses; analysis; organic waste; aloe vera.

\section{INTRODUCCIÓN}

La sábila (Aloe vera) es una planta del género Aloe perteneciente a la familia Liliaceae, originario de África, aunque otros autores lo ubican en África nororiental, Sudáfrica y América central (Gómez et al., 2001). Es una hierba carnosa de 50 a $70 \mathrm{~cm}$ de altura, con hojas agrupadas, tallos de aproximadamente 30 a $40 \mathrm{~cm}$ de longitud, con el borde espinoso; sus flores son tubulares, colgantes, rojas y sus frutos son capsulares. Existen más de 180 especies de sábila, sin embargo, a la más utilizada por sus beneficios se le ha denominado Aloe Vulgaris. Es una planta a la que se le ha encontrado una gran utilidad como cultivo y como producto para la salud (Atherton, 1998).

La sábila o aloe, también llamado áloe, sábila (igual que la ya nombrada) o Aloë, es un género de plantas suculentas de la familia Asphodelaceae con alrededor de 400 especies. Es nativo de las regiones secas de África y Oriente Medio, aunque se haya difundido en todo el mundo en la actualidad (Acudelca, 2006). Su nombre viene del griego aloê; en árabe alloeh, en chino aloehei. No debe confundirse con el agave una planta parecida pero que, debido a las clasificaciones botánicas, pertenece a la familia de los amarilidáceos (Vega et al., 2005).

El Aloe Vera durante siglos fue utilizada por sus propiedades medicinales y terapéuticas sin ningún entendimiento claro o análisis científico de cada una de sus propiedades. En la actualidad, se usa en muchos lugares del mundo, en la medicina moderna para tratar múltiples enfermedades además de ser utilizada en la industria cosmetológica, farmacéutica y alimentaria. Se le atribuyen también efectos tales como, antihemorroidal, cicatrizante, anticatarral, colerética, antitumoral y antiblenorrágica (Martínez y Betancourt, 2000).

Colombia tiene potencial para ser un gran productor y comercializador de la planta, sin embargo, el mercado de sábila es relativamente joven, debido en gran parte a la poca iniciativa y cooperación entre sector público y privado (Figueredo

y Morales, 2010), sin embargo, este crecimiento porcentual de la siembra no va de la mano con su aprovechamiento industrial pues este no ha tenido un desarrollo significativo (Torres, 2013)

Actualmente continúan las investigaciones de las distintas propiedades farmacológicas que se le atribuyen al gel de Aloe Vera, pero no se ha investigado sobre los posibles usos que se le pueden dar a los residuos sólidos orgánicos generados en los procesos agroindustriales del gel del Aloe Vera.

Investigadores como Hernández y Giraldo (2011) realizaron el estudio bromatológico y microbiológico al mucilago de Aloe vera y fertilidad de los suelos de cultivos de los municipios de Guática y Mistrató del departamento de Risaralda, la investigación consistió en realizar un estudio bromatológico, microbiológico al mucilago, foliar y fertilidad de los suelos, en los cultivos de Aloe, se analizaron las condiciones del cultivo, la calidad y tipo de suelo. Los análisis consistieron en determinar parámetros como: humedad, ceni- 
zas, metales y minerales en el mucilago, además se determinó materia orgánica, $\mathrm{pH}$, Potasio $(\mathrm{K})$, Calcio (Ca), Magnesio (Mg), Fosforo (P), Hierro $(\mathrm{Fe})$, Manganeso $(\mathrm{Mn})$, Zinc $(\mathrm{Zn})$, y Cobre $(\mathrm{Cu})$ en el suelo. En cuanto a los análisis microbiológicos realizados, se llevaron a cabo con el fin de estimar la cantidad de aerobios mesófilos, Mohos y levaduras presentes en el mucilago del Aloe vera. De igual forma se observó la ausencia de microorganismos patógenos como: Pseudomona aeroginosa, Staphylococcus aureus, Salmonella sp y coliformes fecales.

Por su parte Figueredo y Morales (2010) elaboraron el plan integral para la comercialización de Aloe vera en Colombia, dando a conocer los principales elementos a tener en cuenta para elaborar un proyecto que explore las diferentes actividades de cultivo, transformación y comercialización de extractos de Aloe vera.

Los residuos agroindustriales componen una fuente importante de azúcares, almidones y materiales celulósicos. Para que se puedan utilizar en las dietas de animales se pueden someter a métodos químicos que ajusten o mejoren su composición nutricional al aumentarle su contenido de proteína y digestibilidad con la acción de microorganismos, entre los que destacan los hongos (Gómez et al., 2013)

\section{METODOLOGÍA}

\section{Clasificación de los residuos orgánicos biode- gradables.}

Se empleó la clasificación de residuos sólidos biodegradables (puntas, cascaras y bordes espinosos) establecida en el Reglamento Técnico para el sector de agua potable y saneamiento básico- Ras (2012).

\section{Muestreo}

El primer paso es recolectar la muestra de los desechos orgánicos (espinas, puntas y cascara) de la sábila que genera la planta de procesamiento que se encuentra ubicada en el municipio de Montenegro, departamento del Quindío, geo referencialmente a $4^{\circ} 34^{\prime}$ de latitud Norte, y $75^{\circ} 45^{\prime}$ de longitud al oeste de Greenwich. Su altura sobre el nivel del mar es de 1294 metros, con una temperatura promedio de $21^{\circ} \mathrm{C}$.

\section{Análisis Bromatológico}

Este análisis se realizó por triplicado en las instalaciones del LIP (Laboratorio de Investigaciones en Poscosecha) de la planta piloto en la Facultad de Ciencias Agroindustriales de la Universidad del Quindío, siguiendo las metodologías oficiales de la Norma Técnica Colombiana (NTC), tabla 1.

Tabla 1. Análisis Bromatológico de los residuos orgánicos del Aloe vera

\begin{tabular}{|c|c|}
\hline PRUEBA & METODOLOGÍA \\
\hline Humedad & NTC 4888 \\
\hline Cenizas & NTC 4648 \\
\hline Grasa & NTC 668 \\
\hline Fibra & NTC 668 \\
\hline Actividad de Agua & Aqualab \\
\hline pH & NTC 440 \\
\hline
\end{tabular}

\section{RESULTADOS Y DISCUSIÓN}

En la Tabla 2, se muestran los porcentajes obtenidos en cada una de los análisis bromatológicos realizados a los residuos de sábila.

Tabla 2. Análisis bromatológico a residuos orgánicos de Aloe vera de la Empresa Sabimax Loe del Municipio de Montenegro.

\begin{tabular}{|c|c|c|}
\hline ANALISIS & $\%$ & NORMA \\
\hline Humedad & $92,4 \pm 0,2$ & NTC 4888 \\
\hline Cenizas & $0,43 \pm 0,01$ & NTC 4648 \\
\hline Grasa & $1,25 \pm 0,04$ & NTC 668 \\
\hline Fibra & $39,6 \pm 0,1$ & NTC 668 \\
\hline $\begin{array}{c}\text { Actividad de } \\
\text { agua }\end{array}$ & $0,95 \pm 0,1$ & Aqualab \\
\hline pH & $4,98 \pm 0,02$ & NTC 440 \\
\hline
\end{tabular}




\section{Contenido de humedad}

Como se aprecia en la tabla 2, el contenido de humedad en los residuos orgánicos de Aloe vera procesada en la planta en base húmeda se encuentra en un $\mathbf{9 2 , 4 \%}$, encontrándose un porcentaje por debajo de los datos teóricos $\mathbf{9 8 , 5 \%}$, esto puede ser a que los datos teóricos hacen relación a las pencas u hojas de la sábila, las cuales tiene mayor cantidad de agua mientras que la mezcla de los residuos de sábila (puntas, cascaras y bordes espinosos) no contiene mucha cantidad de humedad.

Según Restrepo et al. (2010), el porcentaje restante de humedad corresponde a materia sólida que contiene una serie de compuestos, como vitaminas hidrosolubles y liposolubles, minerales, enzimas, polisacáridos, compuestos fenólicos y ácidos orgánicos.

\section{Contenido de cenizas}

Como se puede observar en la tabla 2, el contenido de cenizas en los residuos orgánicos del Aloe vera presenta un $(\mathbf{0 , 4 3 \% )}$ encontrándose dentro del rango propuesto por los datos teóricos. Esto puede indicar que el contenido de cenizas, está relacionado directamente con un alto contenido en minerales que sufren procesos de combustión o quemazón. Wani et al. (2010) en una caracterización que menciona de la planta, muestra que los minerales presentes son: sodio, potasio, calcio, magnesio, cobre, zinc, cromo, y hierro.

Se trata de un parámetro dependiente del contenido de minerales presentes en la corteza que, a su vez, depende de las circunstancias del terreno donde se cultiva esta planta (Zúñiga y Gutiérrez, 2010).

\section{Contenido de fibra.}

Los contenidos de fibra en los residuos orgánicos del Aloe presentan un $\mathbf{3 9 , 6 \%}$, encontrándose una notoria diferencia entre el análisis de los residuos sólidos con respecto a los datos teóricos, esto se debe a que en los datos teóricos se realizan con el mucilago de la sábila.

\section{Contenido de extracto etéreo o grasa}

Como se observa en la tabla 2, el contenido de grasa o extracto etéreo en el residuo sólido orgánico proveniente de los procesos agroindustriales de Aloe vera en la planta es de $\mathbf{1 , 2 5} \%$, encontrándose un ligero incremento en el porcentaje de grasa con respecto a los datos teóricos (Hernández y Giraldo, 2011)

El extracto etéreo se entiende como el contenido lipídico que presenta la cáscara de la sábila. El porcentaje encontrado resultó levemente superior al referido por Paredes et al. (2010). Se trata de un indicador que influye positivamente al momento de someter estos residuales a fermentaciones microbianas para mejorar sus cualidades como alimentos de uso animal; en particular cuando se emplean hongos (Prescot et al., 1999)

No se observa una diferencia significativa entre el contenido de grasa de los residuos orgánicos con el gel, esto indica que la apariencia física no estaría relacionada con el contenido de grasa del gel. Además, los aceites grasos son muy ubicuos en el organismo vegetal pero aun así representan una proporción muy pequeña de su peso en seco.

\section{pH}

Según la tabla 2, la muestra presenta un $\mathrm{pH}$ de 4,98 por lo cual se encuentra dentro del rango establecido por los datos teóricos $(3,5-6,5)$; esto indica que posiblemente el suelo donde está sembrada el cultivo es un suelo acido.

\section{Actividad de agua (Aw)}

El valor obtenido para para la prueba de actividad de agua equivale a $0,95 \%$ y como no se tiene datos teóricos de esta actividad para residuos sólidos orgánicos no se puede hacer una comparación, pero este alto porcentaje de actividad de agua indica que tiene agua libre y ligada. Dicho 
valor de Aw es conforme y relacionada con la obtenida por Vega et al., (2011), en el gel de sábila ya que esta muestra una actividad de agua mayor a $0.98 \%$, lo cual revela que es susceptible al deterioro microbiano, así como a reacciones enzimáticas y de oxidación.

En síntesis, los residuos sólidos orgánicos del Aloe vera, alcanzaron niveles normales, cercanos a los estudios o datos teóricos, en los parámetros de $\mathrm{pH}$, humedad, grasa, actividad de agua y ceniza. Y en el contenido de fibra, fue donde se pudo evidenciar una diferencia notoria.

\section{CONCLUSIONES}

En el análisis bromatológico realizado a los resi- duos sólidos orgánicos generados con el proceso agroindustrial del Aloe vera en la empresa Sabimax Loe se determinó que este contiene 92,4\% de humedad, $0,43 \%$ de cenizas, $39.6 \%$ de fibra, $1,25 \%$ de extracto etéreo, $0.95 \%$ de actividad de agua y un $\mathrm{pH}$ de 4,98. Por lo que se encontraron en un nivel promedio o dentro de los rangos teóricos, aunque tuvo un alto contenido en fibra.

De acuerdo a los análisis bromatológicos realizados a los desechos orgánicos y el alto porcentaje de fibra que arrojo el análisis de laboratorio este tipo de residuos podría usarse como complemento alimenticio para bovinos y especies menores.

\section{BIBLIOGRAFIA}

1. ACUDELCA. (2006). Colombia en el contexto mundial de la producción del aloe vera. Bogotá: Bruguera.

2. Atherton P., Ramos I. Efecto antiulceroso de fórmulas que contienen extracto de Aloe. (1996). Revista Cubana Plant Med.

3. Figueredo C.a., Morales J, C (2010). Plan Integral Para La Comercialización De Aloe Vera En Colombia (tesis de Pregrado). Universidad del Rosario. Bogotá, Colombia. http://repository.urosario.edu.co/

4. Gómez, J.; Yepes, S Y Barahona, R. (2013). Caracterización nutricional del residuo del cultivo de la seta Agaricus bisporus como alimento potencial para bovinos. Revista CES Medicina Veterinaria y Zootecnia, 8 (1), 37-59. https://doi.org/10.21615/2832

5. Gómez, L. F., E. L. Vivas, et al. (2001). "Prácticas de cultivo y algunos factores edafológicos que podrían influir sobre la calidad del gel de sábila.” Rev Chapingo, Serie Zonas Áridas. II (2): 68-73.

6. Hernández Gutiérrez, J.e., Giraldo, J.d. (2011). Estudio bromatológico y microbiológico al mucilago de aloe vera y fertilidad de los suelos de cultivos de los municipios de Guática y Mistrató del departamento de Risaralda. Universidad Tecnológica de Pereira.

7. Martínez M. J., Betancourt B. J. (1996). Ausencia de actividad antimicrobiana de un extracto acuoso liofilizado de Aloe vera (SÁBILA). Revista Cubana Plant Med.

8. Paredes, D.; Álvarez, M. Y Silva, M. (2010). Obtención de Enzimas Celulasas por Fermentación Sólida de Hon-gos para ser Utilizadas en el Proceso de Obtención de Bioalcohol de Residuos del Cultivo de Banano. Revista Tecnológica ESPOL-RTE. 23 (1): 81-88.

9. Prescott, L.; Harley, J. Y Klein, D. (1999). Microbiología (4ta Edición). España: Mc Graw Hill Interamericanade España.

10. restrepo $\mathrm{f}$, jorge $\mathrm{i}$ and aristizabal $\mathrm{t}$, iván d. CONSERVACIÓN DE FRESA (Fragaria x ananassa Duch cv. Camarosa) MEDIANTE LA APLICACIÓN DE RECUBRIMIENTOS COMESTIBLES DE GEL MUCILAGINOSO DE PENCA SÁBILA (Aloe barbadensis Miller) Y CERA DE CARNAÚBA. Vitae. 2010, 17(3): 252-263.

11. Torres, G. (2013). Cálculo de la resistencia a la penetración en hojas de aloe vera (Barbadenses Miller). Revista Acta Agronómica, 62(4), 289 - 295.

12. VVega G, Antonio, Ampuero C, Nevenka, Díaz N, Luis, \& Lemus M, Roberto. (2005)El Aloe vera (Aloe barbadensis Miller) como componente de alimentos funcionales. Revista chilena de nutrición, 32(3), 208-214. https://dx.doi.org/10.4067/S0717-75182005000300005

13. Vega, G.a., Miranda, M., Aranda, M., Henríquez, K., Vergara, J., Tabilo-Munizaga, G. Y Pérez-Won, M. (2011). Effect of high hydrostatic pressure on functional properties and quality characteristics of 
Aloe vera gel (Aloe barbadensis Miller). Food Chemistry, 129(3), 1060-1065. https://doi.org/10.1016/j. foodchem.2011.05.074

14. Wani Y., N. Hasan, M. A. Malik. (2010). Chitosan and Aloe Vera: Two Gifts of Nature. Journal of Dispersion Science and Technology, 31(6), 799-811, DOI: https://doi.org/10.1080/01932690903333606.

15. Zuñiga, P. Y Gutiérrez, R. (2010). Evaluación nutrimental del residuo obtenido de la molienda Húmeda de ceba-da. XII Congreso Nacional de Ciencia y Tecnología de Alimentos, Universidad de Guanajuato, México. 